\title{
4D printing smart biosystems for nanomedicine
}

\author{
Wei Zhu' ${ }^{1}$, Thomas J Webster ${ }^{2}$ \& Lijie G Zhang ${ }^{* 1,3,4,5}$ \\ ${ }^{1}$ Department of Mechanical \& Aerospace Engineering, The George Washington University, Washington, DC 20052, USA \\ ${ }^{2}$ Department of Chemical Engineering, Northeastern University, Boston, MA 02115, USA \\ ${ }^{3}$ Departments of Electrical \& Computer Engineering, The George Washington University, Washington, DC 20052, USA \\ ${ }^{4}$ Department of Biomedical Engineering, The George Washington University, Washington, DC 20052, USA \\ ${ }^{5}$ Department of Medicine, The George Washington University, Washington, DC 20052, USA \\ *Author for correspondence: Tel.: +1 202994 2479; Fax: +1 202994 0238; Igzhang@gwu.edu
}

"Integrating nanomaterials with 4D printing paves new ways toward greater control over the 4D transformation of the printed products to stimulation, and holds great potential in medicine in the development of smart tissue scaffolds, biosensors, bioactuators, biorobots and various other smart medical systems"

First draft submitted: 29 March 2019; Accepted for publication: 16 April 2019; Published online: 12 July 2019

Keywords: 4D printing • biomedical device • nanomaterial • smart material • tissue scaffold

In recent years, 3D printing has been extensively explored in medicine for manufacturing various biomedical products. Referring to 3D printing applications in nanomedicine, it should involve either printing biomedical products in nanoscale resolution or printing nanocomposites [1]. Because of the limitation of current printing techniques, $3 \mathrm{D}$ printing with nanoscale resolution is still challenging. Current $3 \mathrm{D}$ printing in nanomedicine focuses on printing nanocomposites that comprise nanomaterials and host material matrices. With the rapid development of material science and additive manufacturing, $3 \mathrm{D}$ printing has evolved into a new stage $-4 \mathrm{D}$ printing. $4 \mathrm{D}$ printing involves the fourth dimension of time, which confers $3 \mathrm{D}$ printed products with the capacity of deforming upon external stimulation post-printing. 4D printed products are able to change their shape/function on-demand over time, which attracts substantial attention in various research fields including the biomedical field $[2,3]$. Integrating nanomaterials with 4D printing paves new ways toward greater control over the $4 \mathrm{D}$ transformation of the printed products to stimulation, and holds great potential in medicine in the development of smart tissue scaffolds, biosensors, bioactuators, biorobots and various other smart medical systems.

\section{Smart materials \& nanomaterials for 4D printing}

There are two major ways to realize $4 \mathrm{D}$ printing. One is to integrate transforming information in the initial structural design. The typical mechanism is the anisotropic design in a printing process [4]. Under this mechanism, the self-morphing of printed objects can be achieved through precisely controlling the relative orientation and location of heterogeneous ink materials in layered structures [5]. The shape/property transformation of the printed products can be initiated by various stimuli, such as temperature, $\mathrm{pH}$, humidity, stress, electricity, magnetic field, light, acoustics or a combination of these stimuli, and undergo a large variety of possible mechanisms.

The other way to achieve a $4 \mathrm{D}$ effect is to print smart materials which can respond to external stimuli and change their shape. The printable inks can comprise a single smart material, multiple smart materials or a mixture of smart materials with conventional materials. The smartness of printable ink, referring to shape memory, self-adaptability, self-sensing and multiple functionalities, determines how the printed products change their shape/properties in response to the external stimuli [6]. The mechanism under this $4 \mathrm{D}$ transformation is that shape morphing of the printed products results from the change of intrinsic physiochemical properties, such as shape memory $[7,8]$. Shape memory materials can be frozen on a temporal deformation, and then triggered to release the accumulated stresses gradually recovering to the original shape upon heating $[9,10]$. Printing shape memory materials can control the spatial localization of materials to realize gradational, sequential or local shape memory responses. 
Integrating novel smart materials with typical nanomaterials into 3D printing gives rise to a new concept of $4 \mathrm{D}$ printing nanocomposites. Because of their unique physical and chemical properties, nanomaterials may bring a lot of merit to advance $4 \mathrm{D}$ printing. One such merit is that nanomaterials are able to change the shear-thinning properties of bioinks to assist with 4D printing [11]. Shear thinning is a significant property for extrusion-based $3 \mathrm{D} / 4 \mathrm{D}$ printing as high-fidelity structures can be only achieved through reversible changes in the shear viscosity of printable inks. One study has employed a kind of nanoclay, Laponite, in 4D printing in order to improve the shearthinning properties of bioinks. Laponite possesses excellent thixotropy in water which endows the shear-thinning ability of bioinks and self-supported features post-printing [12]. In addition, nanomaterials can also reinforce the mechanical properties of $4 \mathrm{D}$ printed products [12]. Besides, through the use of pristine nanomaterials, nanomaterials can be engineered to have specific characteristics (such as photosensitivity, thermosensitivity and incorporated into printable ink) to improve the stimuli-responsiveness of printed products. In a report by Kim et al. [13], barium titanate nanoparticles were chemically modified with acrylate surface groups and incorporated into printable inks. It was found that the modified nanoparticles can form direct covalent linkages with the polymeric ink under light exposure. The incorporation of modified nanoparticles also resulted in a ten-times increase in piezoelectric coefficients. These results suggest that applying nanomaterials to printable inks would contribute a lot to the application of applying $4 \mathrm{D}$ printing to many high-value research fields.

\section{D printing nano-tissues}

One of the intriguing applications of $4 \mathrm{D}$ printing in medicine is the manufacturing of scaffolds for tissue regeneration. The natural tissue regeneration process usually involves a dynamic change in tissue conformations that may be significant for functional recovery. Even though 3D printing techniques are able to create tissue scaffolds with complex 3D shapes and accurate microarchitectures, it cannot precisely mimic the dynamic nature that occurs in natural tissue repair. With this in regard, $4 \mathrm{D}$ printing has been brought into the field of tissue engineering, which includes stimuli-response effects into 3D printed tissue constructs and provides the possibility to recapitulate the dynamic change in the process of tissue regeneration. Such 4D transformation can offer a traction force for cells that may influence cell phenotype, cell-cell communication and cell density and orientation, and eventually contribute to tissue regeneration.

Considering the nanostructural features of human tissues [14], nanomaterials have been introduced into 4D printing to produce smart nano-bioinks for the creation of tissue scaffolds. The nanomaterials may interact with smart materials to amplifying $4 \mathrm{D}$ effects in printed bio-tissues. Our group recently developed a proof-of-concept $4 \mathrm{D}$ printed brain model using near-infrared light (NIR) responsive nanocomposites to evaluate the capacity for controllable 4D transformation, as well as the feasibility of photothermal stimulation for modulating neural stem cell behaviors [7]. In this study, photothermal graphene nanoplatelets were incorporated into a thermally responsive shape memory polymeric bioink. The graphene can absorb photons from NIR illumination to produce thermal energy which eventually initiates the transformation of printed objects. Compared with directly thermal-triggered shape changing processes, this nanomaterial-assisted thermal-triggered shape change is particularly useful in the generation of tissue scaffolds because the long-wavelength NIR is human benign and can efficiently penetrate into human tissue with no biological harm.

In addition to assisting with producing a cell-favorable stimulation, nanomaterials can also tune the curvature of $4 \mathrm{D}$ printed objects, and therefore facilitate the creation of a complex microarchitecture. In one of our studies to produce a neural conduit, we found that nanomaterials can attenuate the laser energy in stereolithography-based printing and contribute to the regulation of the curvature in 4D transformation [15]. Nano-bioinks containing typical functional nanoparticles, such as iron oxide magnetic nanopowder, carbon nanofiber, hydroxyapatite and graphene, all significantly increased the curvature of the $4 \mathrm{D}$-printed architectures when compared with the pure polymeric ink. Meanwhile, the 4D curvature of printed objects can be tuned by manipulating the nanoparticle concentrations for advancing specific criteria of various applications.

\section{D printing nano-smart biomedical devices}

Combined with the merits of nanomaterials, $4 \mathrm{D}$ printing has tremendous potential in the development of nanosmart biomedical devices, such as biosensors, bioactuators, biorobots, etc. $4 \mathrm{D}$ printed biomedical devices can be designed to monitor various physiological changes, in which nanomaterials could serve as the receptor of external stimulation, enhancer of $4 \mathrm{D}$ shape morphing, supporter of the architecture as well as many others. Possible applications may include utilizing biosensors to detect physical activity of cells and metabolites for diagnostics, 
printing actuators that could actuate through heating or cooling, developing biorobots to deliver therapeutic agents and release upon external stimulation.

\section{Conclusion \& future perspective}

4D printing applies smart materials to manufacture advanced biosystems capable of dynamically changing conformation for a variety of biomedical applications. Even though only a limited number of smart materials have been explored so far for $4 \mathrm{D}$ printing, one can expect a range and variety of printable smart materials that will expand accompanying improvements in printability, controllability and smartness. The integration of smart materials with nanomaterials has emerged as an intriguing area for $4 \mathrm{D}$ printing. The unique interactions between smart materials and nanomaterials could be investigated and used to facilitate the design of novel $4 \mathrm{D}$ printed biomedical products. $4 \mathrm{D}$ printed dynamic tissue scaffolds would be one of the most attractive biomedical applications of 4D printing. However, the influence of deformation in cell fate should be fully investigated to achieve a controllable fashion for inducting bio-tissue development. Despite 4D printing still in its infancy, it undoubtedly holds great potential for various biomedical applications including but not limited to tissue engineering, biorobotics, bioactuation and biosensing.

\section{Financial \& competing interests disclosure}

This work was supported by NSF MME program grant 1642186 and an NIH Director's New Innovator Award 1DP2EB020549-0. The authors have no other relevant affiliations or financial involvement with any organization or entity with a financial interest in or financial conflict with the subject matter or materials discussed in the manuscript apart from those disclosed.

No writing assistance was utilized in the production of this manuscript.

\section{References}

1. Zhu W, Webster TJ, Zhang LG. How can 3D printing be a powerful tool in nanomedicine? Nanomedicine 13(3), 251-253 (2018).

2. Miao S, Castro N, Nowicki M et al. 4D printing of polymeric materials for tissue and organ regeneration. Materials Today 20(10), 577-591 (2017).

3. Gao B, Yang Q, Zhao X, Jin G, Ma Y, Xu F. 4D bioprinting for biomedical applications. Trends Biotechnol. 34(9), 746-756 (2016).

4. Li YC, Zhang YS, Akpek A, Shin SR, Khademhosseini A. 4D bioprinting: the next-generation technology for biofabrication enabled by stimuli-responsive materials. Biofabrication 9(1), 012001 (2016).

5. Gladman AS, Matsumoto EA, Nuzzo RG, Mahadevan L, Lewis JA. Biomimetic 4D printing. Nat. Mater. 15, 413 (2016).

6. Khoo ZX, Teoh JEM, Liu Y et al. 3D printing of smart materials: a review on recent progresses in 4D printing. Virtual Phys. Prototyping 10(3), 103-122 (2015).

7. Cui H, Miao S, Esworthy T et al. A novel near-infrared light responsive 4D printed nanoarchitecture with dynamically and remotely controllable transformation. Nano Res. doi:10.1007/s12274-019-2340-9 (2019) (Epub ahead of print).

8. Miao S, Cui H, Nowicki M et al. Photolithographic-stereolithographic-tandem fabrication of 4D smart scaffolds for improved stem cell cardiomyogenic differentiation. Biofabrication 10(3), 035007 (2018).

9. Miao S, Zhu W, Castro NJ et al. 4D printing smart biomedical scaffolds with novel soybean oil epoxidized acrylate. Scientific Rep. 6, 27226 (2016).

10. Miao S, Zhu W, Castro NJ, Leng J, Zhang LG. Four-dimensional printing hierarchy scaffolds with highly biocompatible smart polymers for tissue engineering applications. Tissue Engin. Part C 22(10), 952-963 (2016).

11. Chimene D, Lennox KK, Kaunas RR, Gaharwar AK. Advanced bioinks for 3D printing: a materials science perspective. Ann. Biomed. Engin. 44(6), 2090-2102 (2016).

12. Guo J, Zhang R, Zhang L, Cao X. 4D printing of robust hydrogels consisted of agarose nanofibers and polyacrylamide. ACS Macro Lett. 7(4), 442-446 (2018).

13. Kim K, Zhu W, Qu X et al. 3D optical printing of piezoelectric nanoparticle-polymer composite materials. ACS Nano 8(10), 9799-9806 (2014).

14. Zhang L, Webster TJ. Nanotechnology and nanomaterials: promises for improved tissue regeneration. Nano Today 4(1), 66-80 (2009).

15. Miao S, Cui H, Nowicki M et al. Stereolithographic 4D bioprinting of multiresponsive architectures for neural engineering. Advan. Biosyst. 2(9), 1800101 (2018). 
